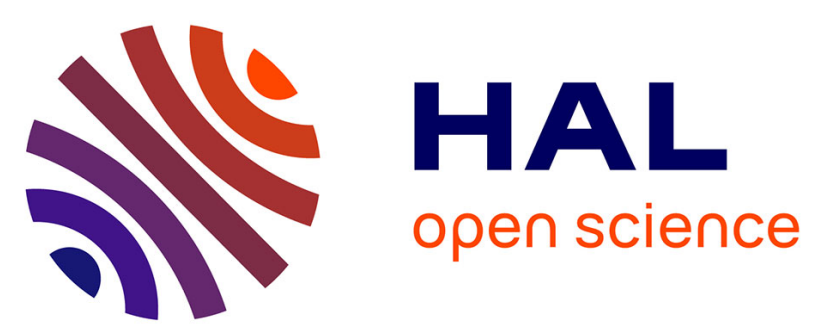

\title{
Transforming Acute Ecotoxicity Data into Chronic Data: A Statistical Method to Better Inform the Radiological Risk for Nonhuman Species
}

Karine Beaugelin, Jacqueline Garnier Laplace, Claire Della-Vedova

\section{- To cite this version:}

Karine Beaugelin, Jacqueline Garnier Laplace, Claire Della-Vedova. Transforming Acute Ecotoxicity Data into Chronic Data: A Statistical Method to Better Inform the Radiological Risk for Nonhuman Species. Environmental Science and Technology, 2020, 211, pp.12376-12382. 10.1021/acs.est.0c03932 . hal-03103033

\section{HAL Id: hal-03103033 \\ https://hal.science/hal-03103033}

Submitted on 7 Jan 2021

HAL is a multi-disciplinary open access archive for the deposit and dissemination of scientific research documents, whether they are published or not. The documents may come from teaching and research institutions in France or abroad, or from public or private research centers.
L'archive ouverte pluridisciplinaire HAL, est destinée au dépôt et à la diffusion de documents scientifiques de niveau recherche, publiés ou non, émanant des établissements d'enseignement et de recherche français ou étrangers, des laboratoires publics ou privés. 
Transforming acute ecotoxicity data into chronic data: a statistical method to better inform radiological risk for non-human species

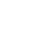

Karine Beaugelin-Seiller ${ }^{\mathrm{a}^{*}}$, Claire Della-Vedova ${ }^{\mathrm{a}, \mathrm{b}}$, Jacqueline Garnier-Laplace ${ }^{\mathrm{a}, \mathrm{c}}$

${ }^{a}$ Institut de Radioprotection et de Sûreté Nucléaire, PSE-ENV, SRTE, St Paul les Durance (France)

${ }^{\mathrm{b}}$ present address EI Claire Della Vedova, Manosque (France)

cpresent address OECD-NEA, Boulogne-Billancourt (France)

*Corresponding author: karine.beaugelin@irsn.fr

Keywords: ecotoxicity, ionizing radiation, acute, chronic, data transformation

Abstract

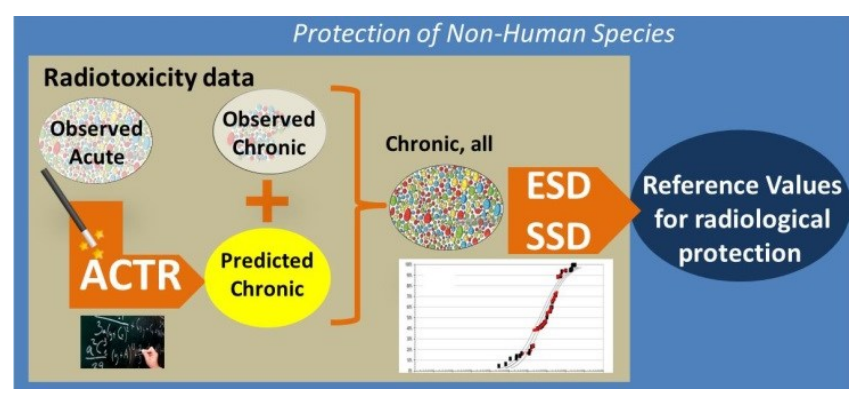

Ecotoxicity data constitute the basic information to support the derivation of ecological benchmark

values, whatever the stressor concerned. However the set of appropriate data may be limited,

especially with regard to chronic exposure conditions. The available data are often biased in favor of acute data from laboratory controlled conditions, much easier to acquire. To make the best use of the available knowledge and better inform effects of ionizing radiation chronic exposure on non-human species, we investigated the transposition to ionizing radiation ecotoxicity of one method proposed for chemicals to extrapolate chronic information from acute toxicity data. Such a method would contribute to enrich chronic data sets required for the derivation of benchmark values making them more robust when used as reference values for ecological risk assessment. We developed accordingly the ACTR (Acute to Chronic Transformation for Radiotoxicity data) approach which we validated. We introduced then the new concept of Endpoint Sensitivity Distribution (ESD). This finally allowed us to compare purely chronic and ACTR-built ESDs for different taxa. For some of them, predicted and observed distributions looked very similar. This promising ACTR method appeared applicable with a 
reasonable level of confidence, but its generalization asks for improvements, some being already identified.

\section{Introduction}

For any ecological risk characterization whatever the exposure situation is, the exposure level(s) of animals and plants need to be compared with some form of numerical criteria which plays the role of benchmark or reference value (concept of reference deviation ${ }^{1,2}$ ). Several methods are internationally recommended $^{3,4}$ for the determination of such reference values, usually depending on the number and nature of available basic ecotoxicity data, such as $\mathrm{ECx}$ (Concentration giving $\mathrm{x} \%$ change in observed effect in comparison with a control). Acquisition of this type of information through laboratory testing has long been subjected to constraints of all kinds, from fundings to ethics. In the field of ecotoxicological research, this situation led to focus on some few model species exposed to a limited set of conditions. As a consequence, the majority of existing work mainly deals with laboratory experiments under a regime of acute exposure to high concentration of toxics, technically much easier to realize and which guarantees a response from exposed organisms. Anyway, the ecotoxicity data today available are mainly acute data, while the main operational needs relate to chronic exposure situations generated by daily human activities. According to this observation, the interest of scientific journals shifted recently towards long term exposure studies, and afferent results become progressively more available, at least for some limited taxa. In the general shared context of resource optimization, using the knowledge accumulated over years about acute toxicity requires developing dedicated approaches.

Looking to derive robust reference values from those experimental outputs relies on the availability of relevant methods. When a chronic ecotoxicity data set is satisfactory in terms of quality and quantity for a given substance, the Species Sensitivity Distribution $\left(\mathrm{SSD}^{5}\right)$ approach is recommended since years now as the best method to determine ecological protection criteria such as EQSs (Environmental Quality Standards $\left.{ }^{3,4}\right)$. However, it can only be used for a small number of substances for which the minimal data set required to build a chronic SSD is met. Considering that at the opposite the set of acute data may be relatively large, methods have been proposed to inform chronic ecotoxicity from 
acute toxicity data: extrapolation ${ }^{6,7}$, Acute to Chronic Ratio ${ }^{8,9}$ (ACR) or Acute to Chronic Transformation $^{10}$ (ACT). Such extrapolation methods are currently applied for example in the framework of Life Cycle Impact Assessments ${ }^{11,12}$ and were implemented in operational tools for a long time by regulatory agencies such as the U.S. EPA ${ }^{13}$ to address data gaps in species sensitivity and reduce reliance on uncertainty factors in ecological risk assessment. Adopting the widely used SSD approach, the ACT approach was developed to transform a data sample assumed to be representative of the acute toxicity of a substance into a sample considered to be representative of the chronic toxicity of the same substance. During the last decade different concepts developed to deal with ecological risk assessment for chemicals have already been successfully transposed to ionizing radiation and radioactive substances ${ }^{14,15,16,17,18}$. This work made it possible in particular to begin to address the issue of effect of stressors mixture on fauna and flora, one key aspect under discussion for regulatory risk assessment. Dealing purely with radiotoxicity eliminates the problem of mixture of radioactive substances as radiotoxic effects expressed as radiological doses are additive.

Adapting and applying such ACT method for radiotoxicity data treatment (ACTR) would expand the chronic dataset from the knowledge related to acute effects, and thereby lead to obtain sufficiently large and qualitative dataset to allow a proper use of statistical extrapolation method such as SSD. This ACT method aims not only to increase the number of available chronic data, but also to enrich qualitatively these data sets. As such, regarding ionizing radiation or radioactive substances, it would make it possible to inform chronic ecotoxicity for species for which there are no experimental chronic data (but only acute ones). The expected increase in the number of data but also in the number of species would give more robustness in the derivation of protection criteria for non-human species exposed to ionizing radiation, the paucity of chronic datasets in terms of quantity and species diversity being identified for a long time as a major weakness of the process.

In this publication, we explore if and how the ACT method may be applied to radionuclides. The related two-phase study is described hereafter, firstly explaining the methodological aspect of the ACTR approach, introducing the concept of Endpoint Sensitivity Distribution (ESD), and then comparing purely chronic and ACTR-built ESDs. 
The ACT for chemicals was developed to establish a relationship between several stressors of the same nature for a single organism. Its transposition to radionuclides implies somewhere a conceptual shift, looking to establish a parallel between multiple taxa for a single stressor, ionizing radiation. Validating the ACTR method will allow to generate new (i.e.predicted) radiotoxicological data from a purely desk study. Such approaches make the best use of already available knowledge and fully comply with the growing demand on ethical and responsible experimentation on living organisms. This is a process in line with the optimization of resources, including the reduction in costs, today expected from all scientist.

\section{Material and methods}

ACT type methods are based on ecotoxicity data acquired for both acute and chronic exposure to a given chemical. In both cases, chemotoxicity is expressed with regard to the chemical concentration in the exposure medium. Biological effects of ionizing radiation, or radiotoxicity, are expressed in terms of dose (rate) that is to say with regard to the energy deposited into the exposed organisms ${ }^{19,20,21}$. Corresponding units are Gy (dose, acute exposure) and Gy per unit of time (dose rate, chronic exposure), that implies significant changes in the methodological approach to convert data from acute to chronic ones, as described later.

\subsection{Radiotoxicitydata}

The FREDERICA database (www.frederica-online.org) in its 2014 updated version ${ }^{22}$ constitutes the primary source of basic radiobiological data for non-human species. Garnier-Laplace et al. (2010) proposed the process of a meta-analysis of these data to build dose (rate)-effect relationships (i.e. doseresponse curves). These curves gave access to parameters analogue to the $\mathrm{EC}_{10}\left(E D R_{10}\right.$, dose rate giving $10 \%$ change in observed effect in comparison with a control - chronic exposure) and $\mathrm{EC}_{50}$ ( $E D_{50}$, dose giving $50 \%$ change in observed effect in comparison with a control - acute exposure). Their standard errors were also determined.

In order to have internally consistent data sets, data from literature reporting observed effects were restricted to external gamma irradiation of non-human species under controlled conditions (either 
laboratory or controlled field) and categorized into acute (high-dose, short term) and chronic (low dose, long term) exposure situations since these exposure regimes lead to different biological or health consequences $^{23}$. This data treatment allowed building a total of about 800 and 240 dose-response relationships for respectively the acute (ca. 135 species) and chronic (ca. 30 species) exposure regime, confirming the large predominance of acute toxicity information.

For comparison, the ACT method is based on three data sources (AQUIRE, the US-EPA database http:/www.epa.gov/ecotox/; a European database - $h \mathrm{ttp}: / / w w w . e c e t o c . o r g /$ and a Dutch report ${ }^{24}$ ). For the 25 substances considered, the number of documented species per substance varied from 5 (12 data) to 133 (977 data) and from 3 (6 data) to 45 (102 data), respectively for acute and chronic exposure regime. These numbers as well as the ratio between acute and chronic information appeared very similar to those characterizing the radiotoxicity data set used to develop the ACTR method.

\subsection{Principles of data transformation}

The proposed ACTR method is intended to infer the parameters of the statistical distribution of chronic radiotoxicity data for a given taxonomic group of organisms (taxon) from the set of observed acute radiotoxicity data available for the same group. More precisely, this statistical approach aims at empirically transforming observed data of acute radiotoxicity $\left(E D_{50}\right)$ into predicted data of chronic radiotoxicity $\left(E D R_{10}\right)$ for any given couple (species, endpoint). The method is inspired from the one published by Duboudin et al. (2004) for chemical substances where ecotoxicity data sets are suffering from similar bias in favor of acute effects data.

The ACTR method consists of a four-step process of statistical modelling. Basically, all observed data $\left(E D R_{10}\right.$ and $\left.E D_{50}\right)$ are first log-transformed to deal with the classical skewness of radiotoxicity data. Mean and standard deviation of the distributions of the two sets of transformed data are determined by taxon (at the class level). Secondly different linear models are tested to predict average chronic distribution parameters from the acute distribution parameters, including simultaneously all the taxa. The best linear models (one model for the mean and one for the standard deviation) are selected. This is performed as part of a process combining a bootstrap with a cross-validation. Thirdly the best 
models are fitted for each taxon to the corresponding observed acute data $\left(E D_{50}\right)$. Applying the fitted models allows finally generating predicted $E D R_{10}$ from the $E D_{50}$ observed for a given taxon.

In details, the taxonomic level of interest is fixed by the identification of sets of observed radiotoxicity data that contain a sufficient number of acute $(E D 50)$ and chronic $(E D R 10)$ data for same groups of organisms (arbitrarily fixed at six data of each type at least to ensure the robustness of the predictions, without constraint on the species number). The level of grouping needed is the taxonomic level to adopt for applying the method. The robustness of the method relies also on the use of consistent radiotoxicity data within each of such a taxon. $E D R_{10}$ and $E D_{50}$ values from the FREDERICA database span several orders of magnitude (respectively nine and height) due to the huge variety of effects reported. For each taxon, extreme values of $E D_{50}$ and $E D R_{10}$ may be assimilated to potential outliers which could bias our analysis. In this context, an outlier is defined as any data which value is outside the range defined by one and a half time the Inter Quartile Interval (note that this factor of 1.5 is usually applied ${ }^{25}$ ). We used a classical univariate detection process, e.g. based on boxplots, to identify outliers in order to eliminate them for the rest of our work.

For each taxon, distribution parameters (mean and standard deviation) of the two sets of observed data are estimated after their log-transformation. The transformation is also convenient for the validation process, allowing the use of the log-normal distribution which is weighted according to species importance. Some species are more or less commonly used for radiotoxicity testing under controlled conditions although no standardized laboratory tests exist, leading to unequal number of observed data per species. To give each species its deserved weight in the data sets, the procedure considered the number of data per species in the data set (acute or chronic) related to a given class. The corresponding weight was calculated applying the equation $1(\mathrm{SI})$ to each class. Once observed $E D R_{10}$ and $E D_{50}$ data selected, class distribution parameters are estimated according to the transformation and weighting procedures using equations 2 to $5(\mathrm{SI})$.

Chronic distribution parameters (mean $w M u C . l g$ and standard deviation $w$ SigmaC.lg) are assumed to be predictable from a linear combination of the acute parameters (mean wMuA.lg and standard 
deviation wSigmaA.lg). The best model is selected among six, what we arbitrarily considered a reasonable number with regard to the number of parameters to be estimated (equations 6 to 11 for the mean and 12 to 17 for the standard deviation, SI). The selection is made by coupling a bootstrap process with a cross validation procedure, and the selected model is the one with the smallest prediction error, averaged at the taxonomic level of interest. The model is used to calculate the chronic distribution parameters predicted for each taxon of this taxonomic level (as illustrated by equations 28 and 29, SI).

Knowing the predicted mean and standard deviation of the chronic distribution, $E D R_{10}$ data can be predicted from observed $E D_{50}$ data. This step requires translating $E D_{50}$ values in units of $E D R_{10}$ values, a procedure called standardization. Observed $\mathrm{ED}_{50}$ data are made dimensionless (Equation 18) and transposed into the chronic dimension (Equation 19). The last step consists in the back-transformation of the result (Equation 20) to obtain the predicted $E D R_{10}$ value.

\subsection{Validation of the ACTR method}

The validation of such a method is typically done by splitting the used data into two subsets, a training one and a testing one. This is possible for sufficiently large data sets, but not for the generally smaller sets usually available for radiotoxicity. The problem may be solved using the same coupling of bootstrap and cross validation previously mentioned, an approach that will allow limiting overfitting.

A first evaluation of the ACTR performances may be obtained visually by comparing for each taxon the ESDs of observed and predicted $E D R_{10}$ data. The ESD is a new concept that corresponds to the socalled $\mathrm{SSD}^{5}$ model restricted to a given taxonomic level and integrating all quality-assessed relevant endpoints per species (those that could lead to changes in population size or structure, i.e. those directly relevant to population demography - mortality, morbidity, reproductive success). It is suggested to build both log-empirical and log-normal ESDs as classically applied to construct SSDs. The log-empirical model is the graphical representation of the empirical cumulative probability of weighted data that implies to weight also this distribution. A modified version of the Hazen method is proposed (Equations 21 to 23, SI). Fitting a log-normal distribution is very common when looking to 
express the statistical distribution of ecological data. The log-normal ESD of predicted $E D R_{10}$ may be plotted by using either the predicted distribution parameters previously defined or, as it was decided here, more accurately recalculating parameters from the predicted $E D R_{10}$ data (Equations 2 to $4, \mathrm{SI}$ ).

Even giving an immediate trend, a visual comparison is always somewhat subjective. A complementary validation procedure is proposed, based on numerical comparisons. $H D R_{5}$ values are estimated per taxon from observed and predicted ESDs using $E D R_{10}$ data. Agreement between these values is estimated from the overlap of their $95 \%$ confidence intervals (see SI, $\S .4$ for calculation details), before to analyze their ratio.

\subsection{Statistics}

All calculations and graphics were done using the version 3.3 .2 of the R language ${ }^{26}$ and already available packages: dplyr $0.7 .6^{27}$; sampling $2.8^{28}$; boot $1.3 .20^{29}$ and ggplot2 $3.0 .0^{30}$ for the graphs.

\section{Results and dis cussion}

The Acute to Chronic Transformation proposed for chemicals has been successfully transposed to ionizing radiation and radioactive substances, taking into account some specificities of the stressor.

The ACTR method deals with a single stressor when the context of the development and application of the original ACT is that of multiple stressors. Additionally the conversion of acute radiotoxicity data into chronic ones implies a change in units $\left(\mathrm{Gy}\right.$ to $\left.\mu \mathrm{Gy} \mathrm{h}^{-1}\right)$. When the ACT for chemicals established a parallel between stressors for a single organism, the innovative aspect of the ACTR method is to look for a parallel between taxa for a single stressor.

\subsection{Data analysis: taxonomic level of interest and identification of outliers}

Applying the size criterion (at least $6 E D_{50}$ and $E D R_{10}$ data per taxon) to the metadata issued from the FREDERICA database (SI) led to identify the class as the lowest taxonomic level for the ACTR implementation (Table 1). From these data, $22 E D R_{10}$ and $20 E D_{50}$ data were identified as outliers (Fig.1) and removed. The Mollusc class, having only four $E D R_{10}$ values (Table 2), was eliminated which finally left nine classes to implement the ACTR method. 
Radiotoxicity data were obtained on species grouped into taxa identified according to their scientific

209

210

211

212

213

name, following the taxonomic habits. At the opposite, uses in the field of ecological risk

characterization are to use common names, which additionally may differ from one to the other

reference consulted. We decided to establish a link between the scientific name of the classes we

considered and common names adapted from those currently employed by the IAEA ${ }^{31}$ and in the

Wildlife Transfer Database. This correspondence has been adopted in order to facilitate to any user aggregation of data at higher levels of taxonomy.

Table 1. the ten classes of potential use for the implementation of the ACTR method

\begin{tabular}{ll}
\hline Scientific name & Common name \\
\hline Actinopterygii & Fish \\
Aves & Birds \\
Branchiopoda & Small crustaceans \\
Gastropoda & Molluscs \\
Magnoliopsida & Fruits and vegetables \\
Malacostraca & Large crustaceans \\
Mammalia & Mammals \\
Monocots & Cereals and grasses \\
Pinopsida & Trees \\
Polychaeta & Worms \\
\hline
\end{tabular}

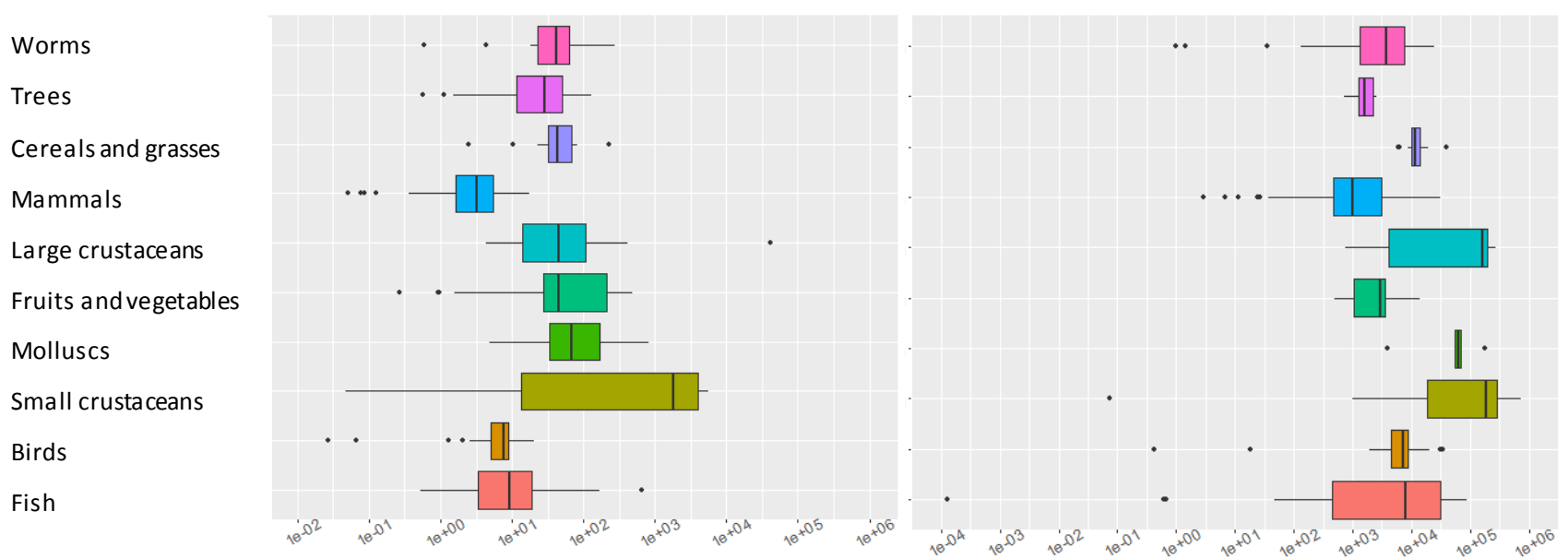

Fig.1. Identification of outliers (data outside the 1.5xInter Quartile Interval) per class in the data set of observed data ( $E D_{50}$ data on the left, $E D R_{10}$ data on the right - x-axis: log of dose or dose rate)

Table 2. Number of radiotoxicity data per class (before outlier detection/after outlier elimination)

\begin{tabular}{lcccc} 
& \multicolumn{2}{c}{$E D_{50}$} & \multicolumn{2}{c}{$E D R_{10}$} \\
\cline { 2 - 5 } Class & Data number & Species number & Data number & Species number \\
\hline Fish & $74 / 73$ & $11 / 11$ & $31 / 28$ & $4 / 4$ \\
Birds & $37 / 33$ & $11 / 11$ & $27 / 23$ & $2 / 2$ \\
Small crustaceans & $15 / 15$ & $1 / 1$ & $9 / 8$ & $2 / 2$ \\
Molluscs & $20 / 20$ & $5 / 5$ & $6 / 4$ & $1 / 1$ \\
Fruits and vegetables & $48 / 45$ & $11 / 10$ & $13 / 13$ & $4 / 4$
\end{tabular}




\begin{tabular}{llrrr} 
Large crustaceans & $19 / 18$ & $6 / 6$ & $7 / 7$ & $2 / 2$ \\
Mammals & $80 / 76$ & $4 / 4$ & $63 / 56$ & $5 / 5$ \\
Cereals and grasses & $17 / 14$ & $6 / 4$ & $30 / 28$ & $2 / 2$ \\
Trees & $35 / 33$ & $5 / 5$ & $8 / 8$ & $3 / 3$ \\
Worms & $13 / 11$ & $1 / 1$ & $27 / 24$ & $2 / 2$ \\
\hline
\end{tabular}

Grey line: class eliminated from the selection after removing outliers due to the too small number of remaining data (below 6)

\subsection{Selection and fit of the model to predict chronic distribution parameters}

Distribution parameters were calculated for the nine classes on observed data, for both acute and chronic data sets (Table SI.1). The best models for predicting the chronic mean and standard deviation were respectively identified as Equations 7 and 12 (SI). Coefficients of these equations were fitted on the whole set of observed acute and chronic distribution parameters, resulting in Equations 28 and 29. Their application to each class generated the parameters of the predicted chronic distribution (Table SI.2).

\subsection{Prediction of $E D R_{10}$ values from $E D_{50}$ data}

The ACTR method has no other ambition than to offer a pragmatic way of transforming acute data into chronic data, with the most "fit for purpose" approach. Therefore we only discuss the quality of the mathematical representativeness of the results obtained.

The $E D R_{10}$ values predicted by applying the ACTR method to the observed $E D_{50}$ data available per class are presented as all other data in the attached Excel® file (SI). The whole set of $E D R_{10}$ data was used to build ESDs per class, fitting both a log-empirical and a log-normal distribution to the observed and predicted data (Fig.2). Parameters of the log-normal distribution were re-calculated, to improve the accuracy of the fitting process (Table SI.3). 

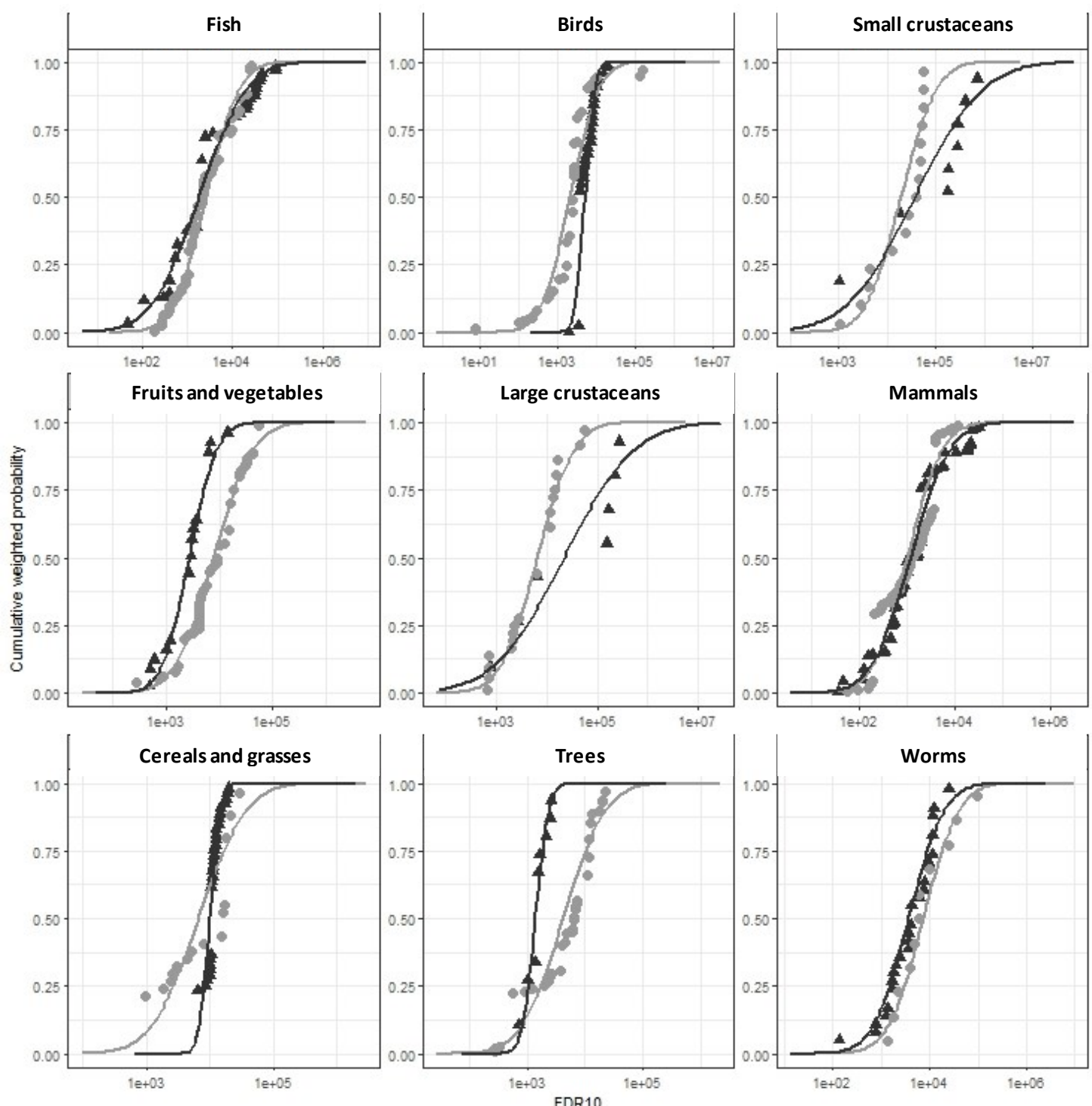

Fig.2. Log-empirical (symbols) and log-normal (lines) ESDs at the class level for observed (black) and predicted (grey) $E D R_{10}$ data (x-axis: $\mu \mathrm{Gy} \mathrm{h}^{-1}, \mathrm{y}$-axis: dimensionless)

Predicted and observed ESDs look very similar for some classes (Fish, Mammals, Worms) but much

less for others (cereals and grasses, crustaceans).

To deeper analyze the ACTR results, $H D R_{5}$ values from observed and predicted ESDs were calculated

(Table SI.4). Error values added to predicted parameters were obtained from the log-normal

distributions fitted with a mean of zero and the standard deviation of residuals, i.e. 0.371 for the mean and 0.377 for the standard deviation. There is a good overlap of $95 \%$ confidence intervals of observed and predicted $H D R_{5}$ values (Fig.3). For most classes of organisms, the range of predicted values encompasses the variation of observed data. For six of the classes (i.e. 67\%), the predicted $H D R_{5}$ value 
Cereals and grasses as well as Birds, the predicted $H D R_{5}$ value is lower than the lower bound of the interval of observed data, which shows that the ACTR approach is conservative. There is finally only one case for which the comparison does not meet the expectation. The $H D R_{5 \_a c t r}$ value predicted for Fish is close to, but higher, the upper bound of the observed data. However, the ratio $H D R_{5 \_a c t r}$ $/ H D R_{S_{-} \text {obs }}$ is about 4 for Fish as for the small crustaceans (Table SI.4), for which the ACTR method seems to give good results. The uncertainty introduced by the prediction is similar, whatever the relative location of the predicted value with regard to the interval of observed data. It should be acknowledged that this ratio for $H D R_{5}$ (i.e. the prediction is 4 times higher than the observation) is the highest of all those calculated.

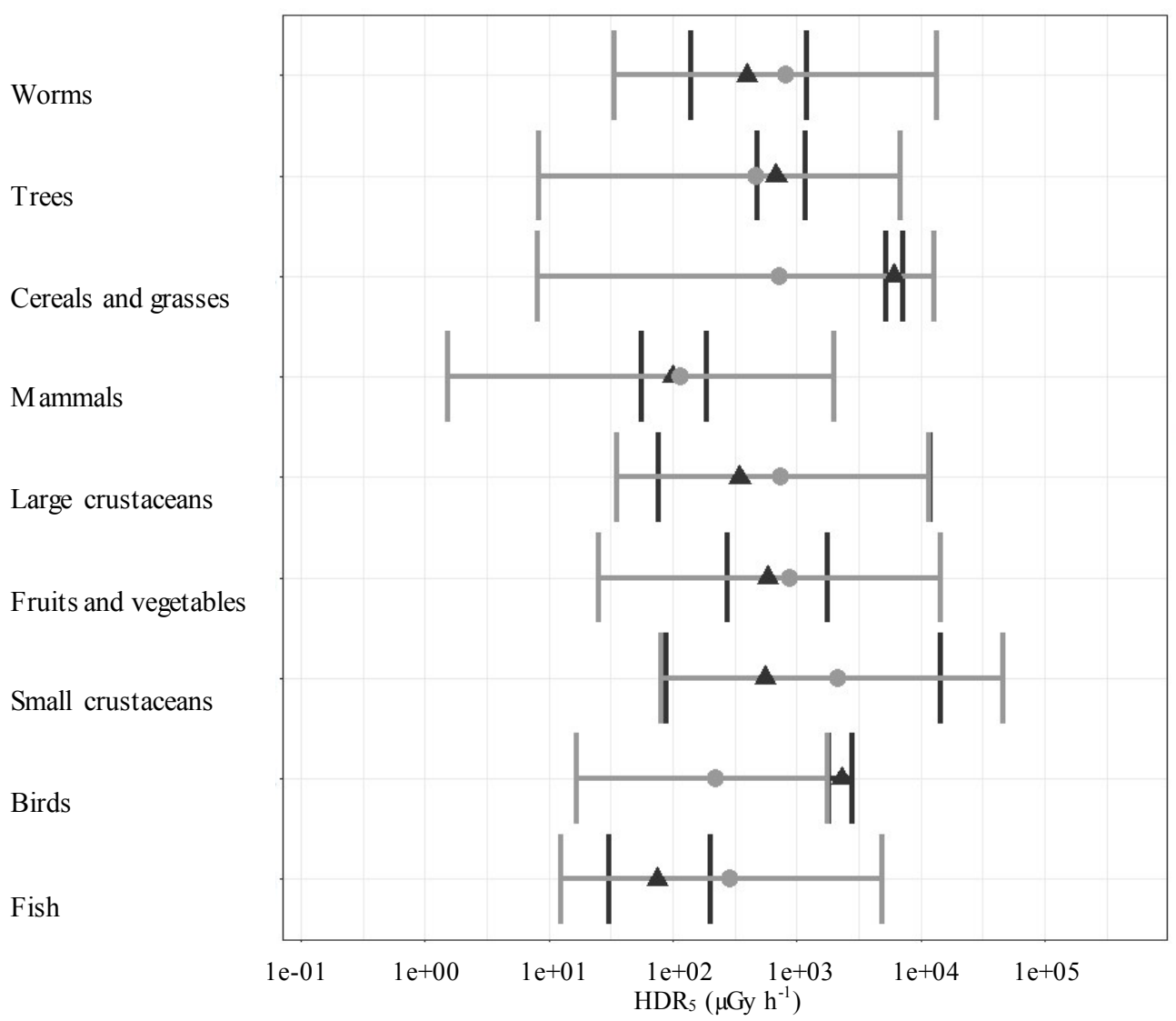

Fig. 3. Overlap between $95 \%$ confidence intervals (lines) of observed (black triangle) and predicted (grey dot) $\mathrm{HDR}_{5}$ with their confidence intervals

Both validation processes gave the same general trend. The numerical comparisons of $H D R_{5_{-} a c t r}$ and $H D R_{5_{-} \text {obs }}$ values confirm the visual comparison of the $E D R_{10}$ distributions (Fig.2) and argue in favor of the ACTR method. 
267

268

269

270

271

272

273

274

275

276

277

278

279

280

281

282

283

284

285

286

287

288

289

290

291

292

The ACTR method appeared promising and seemed to be applicable with a reasonable level of confidence. More precisely, the application of this method to radiotoxicity data led to enrich the observed chronic data set by a factor of two at least for Fish and Mammals. Even more interesting, it allowed increasing also the number of species represented in these enlarged data sets. Such a gain is highly valuable for example to derive protection criteria, as it may permit to move from the very conservative safety factor method to a more realistic statistical approach like the ESD and SSD. In addition to the realism brought by these approaches, the associated transparency should be emphasized. All the available data can be visualized, those taken into account as those identified as outliers and consequently discarded from the treatment. A third benefit of using transparent statistical treatments is the possibility of a continued improvement of their results by introducing new data as they become available. Nevertheless, its generalization comes up against three limitations. Firstly, removing radiotoxicity data identified as outliers leads to ignore the information they contain and can skew the relationship formalizing the ACTR approach. Secondly, the choice of fitting a log-normal distribution as the "right" cumulated probability function was made a priori, without posterior testing. Lastly, distribution parameters had been estimated from small data sets (less than 10) that could be considered insufficient to obtain unbiased estimates.

Our study provided a first brick in the demonstration of the concept of ACT-R that deserves to be more robustly supported. The empirical relationship established here relies on sets of data that could be improved by more research. The ideal data set would include both acute and chronic radiotoxicity data acquired on a same species in the same experimental conditions and, in a perfect world, by the same research team for a sufficient number of representative species. This will certainly not happen, due to too many obstacles on this path (ethic, economic, logistic...). But any new complementary radiotoxicity data will help at least to strengthen the predictive power of our empirical approach that, as any palliative method, will never totally replace experimental acquisition of knowledge.

\section{Acknowledgements}


The work presented in this publication has been performed to support the development of the programme of activities of the Task Group 99 named "Reference Animals and Plants (RAPs) monographs" of the International Committee of Radiological Protection (http://www2.icrp.org/icrp_group.asp?id=92). All members of TG 99 are sincerely thanked for their fruitful contribution to all the related discussions.

\section{References}

1. Official Journal of the European Communities, 2000. Directive 2000/60/EC of the European Parliament and of the Council of 23 October 2000 establishing a framework for Community action in the field of water policy. OJ L 327: 1-73.

2. Jensen J, Mesman M, eds, 2006. Ecological Risk Assessment of Contaminated Land. Decision support for site specific investigations RIVM report number 711701047, $138 \mathrm{p}$.

3. European Commission. 2003. Technical Guidance Document in support of Commission Directive 93/67/EEC on Risk Assessment for new notified substances, Commission Regulation (EC) No1488/94 on Risk Assessment for existing substances, and Directive 98/8/EC of the European Parliament and of the Council concerning the placing of biocidal products on the market. Part II, 2nd ed. Luxembourg, Luxembourg

4. European Commission. 2011. Technical Guidance For Deriving Environmental Quality Standards. Guidance Document No27. Technical Report 2011-055, Common Implementation Strategy for the Water Framework Directive, Luxembourg, Luxembourg

5. Posthuma L II, Suter GWII, Traas T, eds. 2002. Species Sensitivity Distributions in Ecotoxicology. Lewis, Boca Raton, FL, USA.

6. Mayer FL, Krause GF, Buckler DR, Ellersieck MR, Gunhee L.1994. Predicting chronic lethality of chemicals to fishes from acute toxicity test data: Concepts and linear regression analysis. Environ Toxicol Chem 13:671-678.

7. Sun K, Krause GF, Mayer FL, Ellersieck MR, Basu AP. 1995. Predicting chronic lethality of chemicals to fishes from acute toxicity test data: Theory of accelerated life testing. Environ Toxicol Chem 14:1745-1752. 
8. Kenaga EE. 1982. Predictability of chronic toxicity from acute toxicity of chemicals in fish and invertebrates. Environ Toxicol Chem 1:347-358.

9. Parkurst BR, Warren-Hicks W, Cardwell RD, Volosin J, Etchison T, Butscher JB, Covington SM. 1995. Risk managing methods. Water Environ Technol 7:39-43.

10. Duboudin C, Ciffroy P, Magaud H. 2004. Acute-To-Chronic Species Sensitivity Distribution Extrapolation. Environ Tox Chem 23: 1774-1785

11. Rosenbaum RK, Bachmann TM, Gold LS, Huijbregts MAJ, Jolliet O, Juraske R, Koehler A, Larsen HF, MacLeod M, Margni MD, McKone TE, Payet J, Schuhmacher M, van de Meent D, Hauschild MZ (2008). USEtox - The UNEP-SETAC toxicity model: Recommended characterisation factors for human toxicity and freshwater ecotoxicity in life cycle impact assessment. Int J Life Cycle Assess 13:532-546.

12. Müller N, de Zwart D, Hauschild M, Kijko G, Fantke P (2017). Exploring REACH as potential data source for characterizing ecotoxicity in life cycle assessment. Environ Toxicol Chem 36: 492500

13.U.S. EPA (2003). Acute-to-Chronic Estimation (Ace v 2.0) with Time - Concentration -Effect Models. User Manual and Software. EPA/600/R-03/107, United States Environmental Protection Agency, Office of Research and Development (Washington DC, USA).

14. Hingston, J. L., Copplestone, D., Beresford N.A. and Howard, B.J. (eds.), Hingston, J. L., Andersson, P., Beresford, N.A., Brown, J., Copplestone, D., Garnier-Laplace, J., Howard, B.J., Whitehouse, P. 2007. PROTECT Deliverable 3 A review of approaches to protection of the environment from chemicals and ionizing radiation: Requirements and recommendations for a common framework. Contract Number: 036425 (FI6R)

15.Copplestone, D., Andersson, P., Beresford, N.A., Brown, J., Dysvik, S., Garnier-Laplace, J., Hingston, J., Howard, B.J., Oughton, D., Whitehouse, P. 2009. Protection of the environment from ionizing radiation in a regulatory context (PROTECT): Review of current regulatory approaches to both chemicals and radioactive substances. Radioprotection 44, 5, 881-886. 
16. Garnier-Laplace, J., Della-Vedova, C., Andersson, P., Copplestone, D., Cailes, C., Beresford, N.A., Howard, B. J., Howe, P., Whitehouse, P. 2010. A multi-criteria weight of evidence approach for deriving ecological benchmarks for radioactive substances. J. Radiological Prot., 30, 215-233

17. Beaumelle L, Della-Vedova C, Beaugelin-Seiller K, Garnier-Laplace J, Gilbin R. 2017. Ecological risk assessment of mixtures of radiological and chemical stressors: methodology to implement an msPAF approach. Environ Poll 231: 1421-1432.

18. Beaugelin-Seiller K, Gilbin R, Reygrobellet S, Garnier-Laplace J. 2019. A single indicator of harm for people and ecosystems exposed to stable and radioactive substances. Environ. Poll. 249:560565

19. ICRP, 2007. The 2007 Recommendations of the International Commission on Radiological Protection. ICRP Publication 103. Ann. ICRP 37 (2-4).

20.ICRP, 2008. Environmental Protection - the Concept and Use of Reference Animals and Plants. ICRP Publication 108. Ann. ICRP 38 (4-6).

21.ICRP, 2009. Environmental Protection: Transfer Parameters for Reference Animals and Plants. ICRP Publication 114, Ann. ICRP 39(6)

22. IAEA. 2014a. Modelling of Biota Dose Effects. International Atomic Energy Agency, IAEATECDOC-1737, TECDOC Series, Vienna, Austria

23. United Nations Scientific Committee on the Effects of Atomic Radiation UNSCEAR 2008 Sources and Effects of Ionizing Radiation - Volume II - Effects. Report to the General Assembly, with scientific annexes $\mathrm{C}, \mathrm{D}$ and $\mathrm{E}$.

24. National Institute of Public Health and the Environment in the Netherlands - RIVM (1999). Environmental Risk Limits in the Netherlands. RIVM Report 601640001. Belthoven, The Netherlands.

25. Tukey, J. W. 1977 Exploratory Data Analysis. Section 2C.

26. R Development Core Team, 2016. R: A Language and Environment for Statistical Computing (Vienna: R Foundation for Statistical Computing) http://www.R-project.org.

27. Wickham H, François R, Henry L, Kirill Müller K (2018). dplyr: A Grammar of Data Manipulation. R package version 0.7 .6 . https:/CRAN.R-project.org/package=dplyr 
28. Tillé Y, Matei A. 2006. The sampling package. Software manual, CRAN, http://cran.rproject.org/src/contrib/Descriptions/sampling.html.

29.Davison AC, Hinkley DV (1997). Bootstrap Methods and Their Applications. Cambridge University Press, Cambridge. ISBN 0-521-57391-2, http://statwww.epfl.ch/davison/BMA/.

30. Wickham H (2016). ggplot2: Elegant Graphics for Data Analysis. Springer-Verlag New York.

$$
\text { ISBN 978-3-319-24277-4, https } / / \text { ggplot2.tidyverse.org. }
$$

31.IAEA. 2014b. Handbook of Parameter Values for the Prediction of Radionuclide Transfer to Wildlife. International Atomic Energy Agency, IAEA-TRS-479, Technical Report Series, Vienna, Austria.

(1)

